\title{
Direct Quantitative Comparison of Molecular Responses in Photodamaged Human Skin to Fractionated and Fully Ablative Carbon Dioxide Laser Resurfacing
}

\author{
Jefrrey S. Orringer, MD, Dana L. Sachs, MD, Yuan Shao, PhD, Craig Hammerberg, PhD, \\ Yilei Cui, PhD, John J. Voorhees, MD, FRCP, and Gary J. Fisher, PhD*
}

BACKGROUND Fractionated ablative laser resurfacing has become a widely used treatment modality. Its clinical results are often found to approach those of traditional fully ablative laser resurfacing.

OBJECTIVE To directly compare the molecular changes that result from fractionated and fully ablative carbon dioxide $\left(\mathrm{CO}_{2}\right)$ laser resurfacing in photodamaged human skin.

METHODS AND MATERIALS Photodamaged skin of 34 adult volunteers was focally treated at distinct sites with a fully ablative $\mathrm{CO}_{2}$ laser and a fractionated $\mathrm{CO}_{2}$ laser. Serial skin samples were obtained at baseline and several time points after treatment. Real-time reverse transcriptase polymerase chain reaction technology and immunohistochemistry were used to quantify molecular responses to each type of laser treatment.

RESULTS Fully ablative and fractionated $\mathrm{CO}_{2}$ laser resurfacing induced significant dermal remodeling and collagen induction. After a single treatment, fractionated ablative laser resurfacing resulted in collagen induction that was approximately $40 \%$ to $50 \%$ as pronounced as that induced by fully ablative laser resurfacing.

CONCLUSIONS The fundamental cutaneous responses that result from fully ablative and fractionated carbon dioxide laser resurfacing are similar but differ in magnitude and duration, with the fully ablative procedure inducing relatively greater changes including more pronounced collagen induction. However, the molecular data reported here provide substantial support for fractionated ablative resurfacing as an effective treatment modality for improving skin texture.

This study was supported by the University of Michigan Department of Dermatology Laser Research Fund and by an American Society for Dermatologic Surgery Cutting Edge Research Grant. The authors have indicated no significant interest with commercial supporters.

$\mathrm{T}$ raditional fully ablative laser resurfacing remains a gold standard procedure for improving the texture of photodamaged skin, ${ }^{1}$ but concerns regarding recovery times and the potential for significant complications of this procedure, including scarring and permanent dyspigmentation, have led to the development of alternative treatment approaches. ${ }^{2}$ Several years ago, nonablative procedures were developed that proved to be safer and necessitated significantly shorter down times, but results of these treatments were often modest with respect to altera- tions in skin texture. More recently, the concept of fractionated photothermolysis was introduced. ${ }^{3}$ Fractionated treatments generally involve microscopically small beams of energy applied to the skin with sparing of substantial portions of the total treated surface area. In this way, deeply penetrating columns of laser energy heat the dermis and stimulate matrix remodeling while still allowing for a favorable safety profile and rapid healing. The initial devices in this category were nonablative in nature, but even newer systems have applied ablative laser therapy in a fractionated format.

*All of the authors are affiliated with the Department of Dermatology, School of Medicine, University of Michigan, Ann Arbor, Michigan 
Ablative fractionated laser resurfacing attempts to strike a balance between the brief recovery times of other fractionated procedures and the profound clinical efficacy of traditional fully ablative laser resurfacing. Several reports of the effectiveness of this treatment approach have been published, and the safety profile appears to be generally favorable as well, ${ }^{4-7}$ but detailed quantification of the cutaneous cellular and molecular alterations resulting from this promising treatment modality are lacking. In addition, there have been no direct quantitative comparisons of the molecular changes that result from fractionated ablative laser resurfacing with those that occur after fully ablative laser resurfacing.

Prior work at our institution has demonstrated the ability to quantify cutaneous responses to various aesthetic interventions. ${ }^{8-17}$ Our data suggest that the magnitudes of specific molecular responses are predictive of the clinical efficacy of a given treatment intended to improve the texture of photodamaged skin. Furthermore, data that delineate the degree to which the newer fractionated treatments approach typical results of the gold standard of traditional resurfacing may enhance patient education regarding expected outcomes. We therefore sought to examine, quantify, and directly compare dermal remodeling that results from fractionated and fully ablative carbon dioxide $\left(\mathrm{CO}_{2}\right)$ laser resurfacing in photodamaged human skin.

\section{Materials and Methods}

\section{Volunteer Recruitment and Inclusion and Exclusion Criteria}

The institutional review board of the University of Michigan Medical School approved this study, and written informed consent was obtained from all study subjects before entry into the study. Subjects of either sex and any ethnicity or race were eligible. Adult human volunteers aged 50 to 67 (mean 56.8) with clinically evident photodamage of the forearm skin that was rated as moderate to severe were recruited. Treated patients had Fitzpatrick skin types
I, II, or III. Subjects were recruited from the University of Michigan's Cosmetic Dermatology and Laser Center patient base and from the Department of Dermatology's pool of prior research subjects. Subjects were required to be in good health and demonstrate a willingness and ability to understand and adhere to the requirements of the study protocol. Exclusion criteria included a history of infection of the treated skin, a history of keloid scar formation, a history of allergy to lidocaine or other similar anesthetics, oral retinoid use within 1 year of study entry, lack of adherence to the study protocol, prior laser therapy of the treated skin, and a medical history or concurrent illness that investigators deemed unsafe for study participation. Pregnant or nursing patients were also excluded and a 2-week wash-out period was required for all topical agents being applied to the forearm skin.

\section{Laser Treatment and Skin Biopsies}

Subjects underwent a medical history and a cutaneous physical examination of the forearms. Baseline skin samples (4-mm diameter) were obtained using a punch biopsy technique after local anesthesia was achieved with injection of $1 \%$ lidocaine. Medial and lateral strips of photodamaged dorsal forearm skin free from scars were then focally treated with the fully ablative $\mathrm{CO}_{2}$ laser (Ultrapulse, Lumenis, Santa Clara, CA) or the fractionated $\mathrm{CO}_{2}$ laser (Ultrapulse Encore). A computer-generated randomization determined which device was applied to the medial skin strip and which was used to treat the lateral skin strip for each patient.

Treatment parameters for fully ablative laser resurfacing were $300 \mathrm{~mJ}$; power $60 \mathrm{~W}$; and computer pattern generator settings of shape 3 , size 5 , and density 6 . Two passes were performed, with gentle soaking and removal of thermal debris between passes. Fractionated $\mathrm{CO}_{2}$ laser resurfacing was performed in two passes. During the first pass, the device was placed in the Deep FX mode, and treatment parameters were pattern 2, pulses 1 , size 7 , energy $15 \mathrm{~mJ}$, and density $15 \%$. The second pass was performed with the laser in Active FX mode 
with shape 3, size 5, density 3, and $100 \mathrm{~mJ}$. Laser treatment settings were intended to approximate those that are commonly used in a clinical setting.

Patients were instructed in appropriate wound care, including frequent skin soaks using a water and vinegar mixture and applications of petrolatum. They returned periodically for reevaluation and to provide additional skin samples from treated sites at various time points after the treatment. Tissue obtained was analyzed using immunohistochemistry and real-time reverse transcriptase polymerase chain reaction (RT-PCR) technology to assess dermal matrix changes at the protein and messenger RNA (mRNA) levels, respectively.

\section{RNA Extraction, Reverse Transcription, and Quantitative Real-Time RT-PCR}

Extraction of total RNA from whole skin samples and reverse transcription coupled with quantitative real-time PCR was performed as previously described. ${ }^{18}$ Custom primers and probes were used for matrix metalloproteinase (MMP)-1, MMP-3, MMP-9, type I collagen, type III collagen, and 36B4 (RPLP0, ribosomal protein, large, P0). All other primer-probe sets were validated gene expression assays (TaqMan, Applied Biosystems, Foster City, CA) (assay references available upon request). Results are presented as differences between treated and untreated skin samples normalized to transcript levels of house-keeping gene 36B4.

\section{Immunohistochemistry}

Immunostaining was performed on frozen skin sections ( $7 \mu \mathrm{m}$ thick), using primary antibodies that specifically recognized neutrophil elastase (Dako, Carpinteria, CA), MMP-1, MMP-3, MMP-9, procollagen I (all from Chemicon/Millipore, Billerica, MA), or endothelial cell marker CD31 (BD Pharmingen, San Diego, CA). Antibody-antigen complexes were visualized usingwith a secondary antibodyperoxidase-AEC system (Biogenex, Fremont, CA), as previously described. ${ }^{18}$

\section{Procollagen I Protein Measurement}

Procollagen I protein levels were quantified in skin samples according to an enzyme-linked immunosorbent assay (ELISA) using a commercial kit (Panvera, Madison, WI), and normalized to sample dermal volume, as previously described. ${ }^{17}$

\section{Statistical Analysis}

Differences in mRNA levels of specific genes at specific time points during the study were statistically evaluated using repeated-measures analysis of variance. Normality was assumed because of the sample size. Mauchly's sphericity test was used to validate sphericity. If it was violated, the multivariate Wilks' Lambda test statistic was reported. When significant treatment effects were found, individual pairwise comparisons at each time point were made using a paired $t$-test. Summary data are presented as means and standard errors. An overall alpha level of 0.05 was used to determine statistical significance, and all statistical tests are two-sided. All data were analyzed using SAS version 9.2 (SAS Institute, Inc., Cary, NC).

\section{Results}

Patients tolerated the treatments well. One patient developed mildly greater than expected erythema at the treatment sites that was felt to be inflammatory in nature. The patient was treated prophylactically with a course of oral cephalexin, with complete resolution of the erythema. Most patients developed mild hypopigmentation where treated with the fully ablative $\mathrm{CO}_{2}$ laser, and all patients developed small scars at their biopsy sites, as expected. No additional adverse events, including scarring, infection, delayed wound healing, or dyspigmentation, were noted in treated skin.

We have previously reported that fully ablative $\mathrm{CO}_{2}$ laser resurfacing of photodamaged human skin elicits a highly orchestrated wound healing response consisting of an acute inflammatory phase, reepithelization, and protracted remodeling of the dermal 
extracellular matrix (ECM). ${ }^{13,19}$ Acute inflammation is largely an expression of innate immunity, which involves induction of a complex array of cytokines, chemokines, and antimicrobial peptides that stimulate influx and activation of monocytes and neutrophils. Interluekin-1 beta (IL-1 $\beta$ ) is a primary cytokine. Fully ablative and fractionated laser treatment produced significant increases in IL-1 $\beta$ mRNA levels 1 day after treatment (Figure 1A). Induction was 15 times as great after fully ablative laser resurfacing than after fractionated laser resurfacing. IL-1 $\beta$ mRNA levels remained high for at least 1 week after fully ablative $\mathrm{CO}_{2}$ laser treatment, whereas IL-1 $\beta$ gene expression returned to near pretreatment baseline levels within 3 days after fractionated laser treatment.

The chemokine IL-8 (CXCL8) is a potent neutrophil chemoattractant that plays an important role in innate immunity. Fully ablative and fractionated $\mathrm{CO}_{2}$ treatment significantly induced IL-8 gene expression. Maximal induction, which occurred within 1 day after treatment, was more than 10 times as great (5,736-fold) after fully ablative than after fractionated (490-fold) $\mathrm{CO}_{2}$ laser treatment (Figure 1B). The duration of elevation was also substantially longer after fully ablative resurfacing than after fractionated $\mathrm{CO}_{2}$ laser treatment. IL-8 mRNA levels remained significantly higher for at least 2 weeks after fully ablative $\mathrm{CO}_{2}$ treatment, whereas IL-8 levels returned to baseline within 1 week after fractionated $\mathrm{CO}_{2}$ laser treatment (Figure 1B).

Emerging knowledge has highlighted the important role of antimicrobial peptides in innate immune responses to protect against cutaneous infection. We determined gene expression of two well-characterized antimicrobial peptides (AMP), cathelicidin and beta-defensin 2, after laser treatment. Both AMP were significantly induced after fully ablative and fractionated $\mathrm{CO}_{2}$ treatment. Similar to IL- $1 \beta$ and IL8 gene expression, maximal induction of cathelicidin (Figure 1C) and $\beta$-defensin (Figure 1D) was
(A)

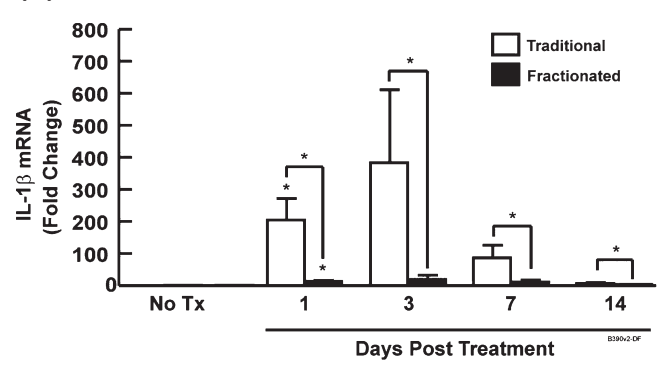

(C)

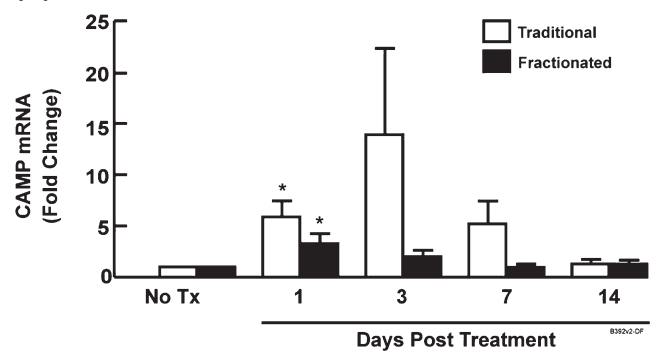

(B)

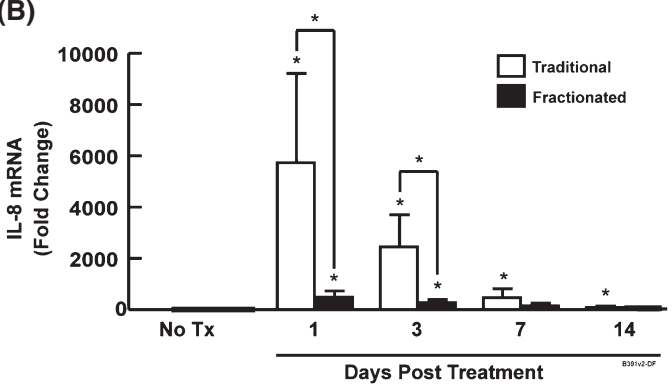

(D)

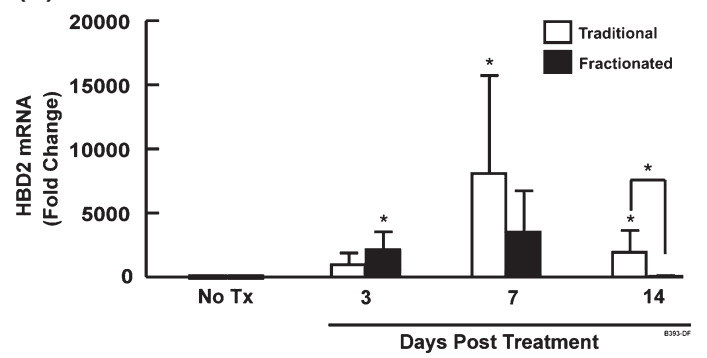

Figure 1. Induction of inflammatory mediators gene expression by fully ablative and fractionated carbon dioxide $\left(\mathrm{CO}_{2}\right)$ lasers in human skin in vivo. Photoaged forearm skin was treated with fully ablative (traditional) or fractionated $\mathrm{CO}_{2}$ laser. Skin samples were obtained at the indicated times after treatment and analyzed using real-time polymerase chain reaction for gene expression of (A) interleukin (IL)-1, (B), IL-8, (C), cathelicidin (CAMP), and (D) human beta definsin-2 (HBD2). Data are means \pm standard errors of the mean, $N=6-10, * p<.05$. 
observed during the first week after treatment, although the magnitude and duration of induction by fully ablative and fractionated $\mathrm{CO}_{2}$ laser treatment did not significantly differ.

Induction of mediators of innate immunity, as described above, causes influx of immune cells into the skin. Neutrophils and macrophages are prominent cell types that participate in the initial stage of wound repair. We assessed the presence of neutrophils and macrophages, identified by the immunohistochemical markers neutrophil elastase and CD68, respectively, in skin after fully ablative or fractionated $\mathrm{CO}_{2}$ laser therapy. Influx of both cells types was observed within 24 hours after treatment. At 3 days, substantial accumulation of neutrophils (Figure 2A) and macrophages (Figure 2B) was observed throughout the dermis in fully ablative $\mathrm{CO}_{2}$ laser-treated skin. Although fractionated $\mathrm{CO}_{2}$ laser treatment also induced influx of neutrophils and macrophages, the extent was substantially less than after fully ablative $\mathrm{CO}_{2}$ laser treatment (Figure 2A, B). By 7 days after treatment, the presence of both cell types was substantially lower in fully ablative and fractionated $\mathrm{CO}_{2}$ laser-treated skin.

In addition to causing immune cell influx, inflammatory mediators induce enzymes that degrade the dermal collagen-rich ECM. This degradation

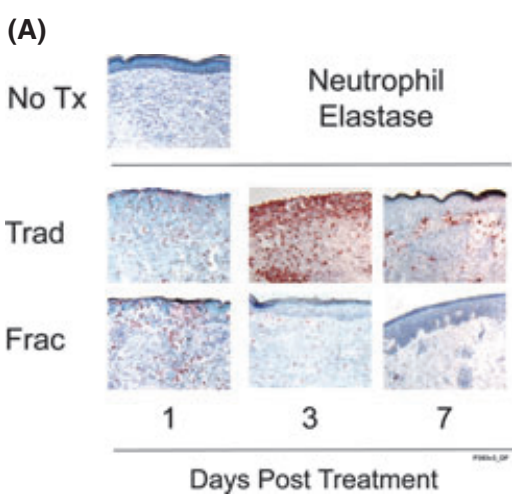

facilitates motility of immune cells within the dermis and sets the stage for production of new ECM during the remodeling phase of wound healing. The enzymes that are primarily responsible for dermal ECM degradation after $\mathrm{CO}_{2}$ laser treatment are MMP-1, MMP-3, and MMP-9. ${ }^{13}$ MMP-1 initiates cleavage of collagen fibrils, which are further degraded by MMP-3 and MMP-9. The mRNA levels of all three MMP were significantly higher within 1 day after fully ablative or fractionated $\mathrm{CO}_{2}$ laser treatment (Figure 3). At this early time point, MMP induction was greater with fractionated laser treatment. Higher MMP production at 1 day after fractionated laser resurfacing reflects the presence of remaining wounded epidermis, which is a rich source of MMP. ${ }^{20}$ At 3 days and at all later time points, fully ablative laser resurfacing induced higher MMP levels than those noted after fractionated $\mathrm{CO}_{2}$ laser treatment (Figure 3). MMP-1 and MMP-3 expression remained high during the first week after treatment and then fell sharply by the second week, whereas MMP-9 expression remained high for at least 2 weeks. The peak mean induction of MMP-1 mRNA 1 week after fully ablative laser treatment $(4,278$-fold) was significantly greater than after fractionated laser treatment (717-fold)

(Figure 3A). Similarly, MMP-3 induction was nearly four times as high after fully ablative resurfacing than after fractionated $\mathrm{CO}_{2}$ laser treatment
(B)

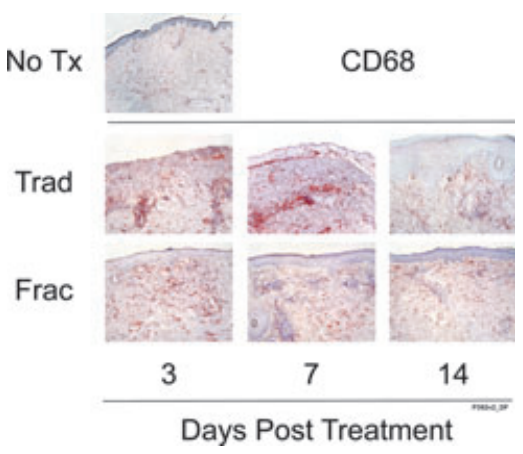

Figure 2. Induction of inflammatory cells using fully ablative and fractionated carbon dioxide $\left(\mathrm{CO}_{2}\right)$ lasers in human skin in vivo. Photoaged forearm skin was treated using fully ablative (traditional) or fractionated $\mathrm{CO}_{2}$ laser. Skin samples were obtained at the indicated times after treatment and analyzed using immunohistology for inflammatory cell markers (A) neutrophil elastase and (B) CD68. Representative images from six subjects are shown. 
(A)

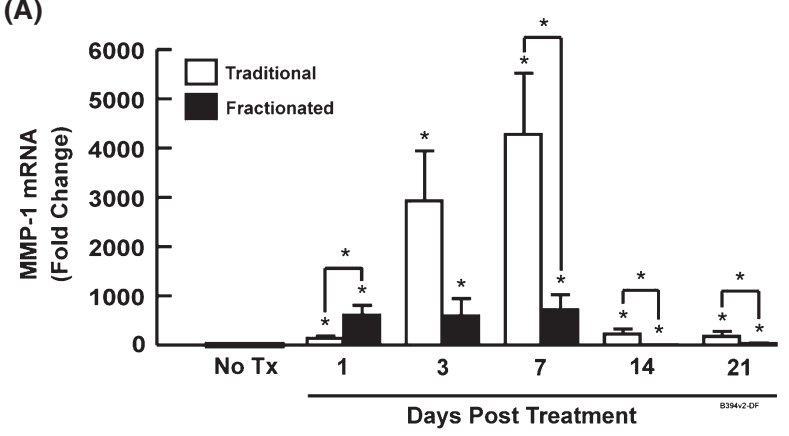

(B)

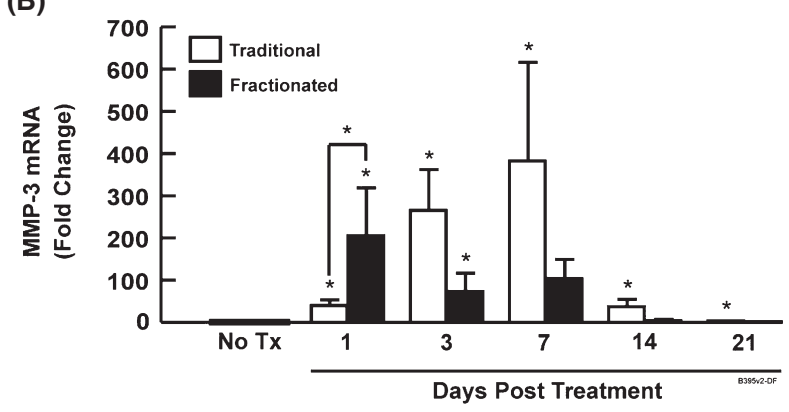

(C)

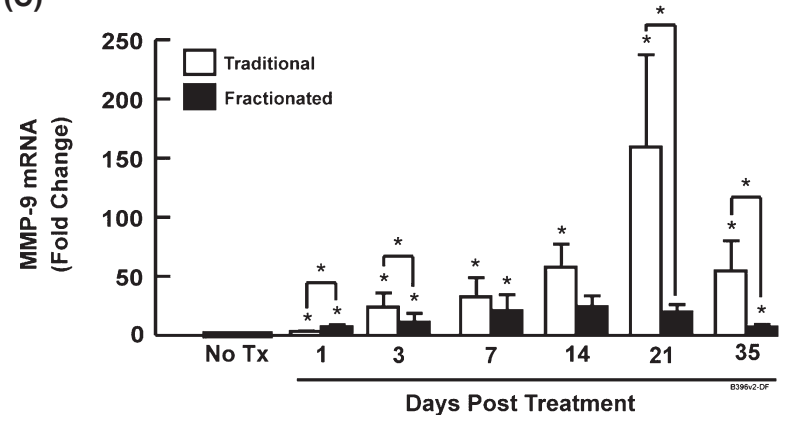

Figure 3. Induction of matrix metalloproteinase (MMP) gene expression using fully ablative and fractionated carbon dioxide $\left(\mathrm{CO}_{2}\right)$ lasers in human skin in vivo. Photoaged forearm skin was treated with fully ablative (traditional) or fractionated $\mathrm{CO}_{2}$ laser. Skin samples were obtained at the indicated times post treatment and analyzed by realtime polymerase chain reaction for gene expression of (A) MMP-1, (B) MMP-3, and (C) MMP-9. Data are means \pm standard errors of the mean, $N=6-10, * p<.05$.

(Figure 3B). MMP-9 gene expression increased in a more delayed fashion. Peak induction of MMP-9 mRNA at 3 weeks after fully ablative laser treatment was six times as great as after fractionated laser resurfacing (Figure 3C).

The temporal pattern of MMP-1 and MMP-3 protein expression after laser treatment was similar to that observed for mRNA induction. Immunostaining for MMP-1 (Figure 4A) and MMP-3 (Figure 4B) was observed throughout the upper dermis. Staining intensity for MMP-1 and MMP-3 was substantially greater after fully ablative $\mathrm{CO}_{2}$ laser treatment than after fractionated $\mathrm{CO}_{2}$ laser treatment. In contrast to MMP-1 and MMP-3, MMP-9 immunostaining differed from its mRNA expression pattern after both types of laser treatment. MMP-9 protein is stored within secretory granules in neutrophils. Influx of neutrophils into skin, as described above, results in a rapid increase in MMP-9 protein levels. Therefore, MMP-9 immunostaining, which largely reflects neutrophilic infiltration, was observed at 1 day after fully ablative or fractionated $\mathrm{CO}_{2}$ laser treatment (Figure 4C). Maximal staining was observed within the first week after treatment, and consistent with neutrophil elastase, staining was substantially stronger after fully ablative than after fractionated $\mathrm{CO}_{2}$ laser treatment. This early induction of MMP-9 protein is largely independent of mRNA induction, which was observed to peak at 2 to 3 weeks after treatment (Figure 3C).

After the inflammatory phase of wound healing, dermal fibroblasts produce new ECM, which is primarily composed of type I and type III collagen fibrils. These collagens are synthesized and secreted as soluble precursors (procollagens), which undergo proteolytic processing to form insoluble collagen fibrils. The mRNA and protein levels of type I and type III procollagen reflect ongoing collagen synthesis. Fully ablative and fractionated laser treatment significantly induced type I procollagen mRNA (Figure 5A) and protein (Figure 5B).

Significant induction of type I procollagen mRNA was observed at 2 weeks and remained high for at least 5 weeks after treatment (Figure 5A) with each laser treatment modality. Average peak induction after fully ablative laser resurfacing (16.4-fold) was almost twice that observed with fractionated $\mathrm{CO}_{2}$ laser treatment (8.4-fold).

Consistent with these mRNA data, immunostaining revealed substantial induction of type I procollagen 
(A)

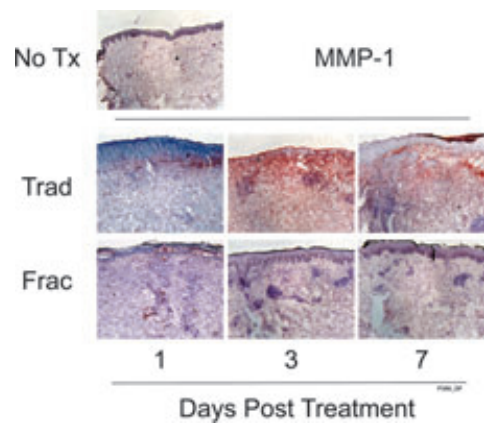

(B)

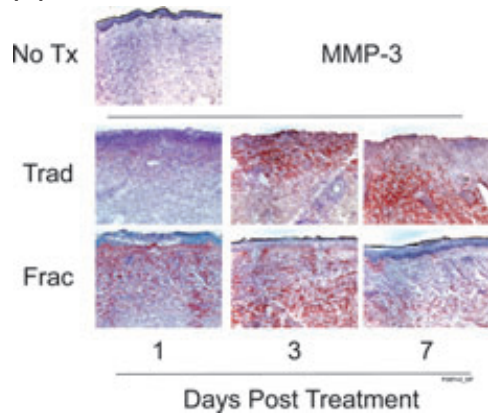

(C)

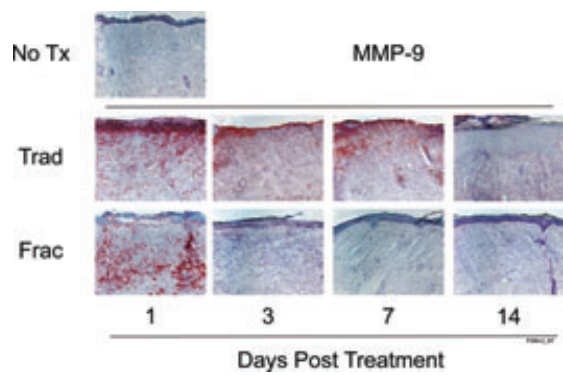

Figure 4. Induction of matrix metalloproteinase proteins (MMP) by fully ablative and fractionated carbon dioxide $\left(\mathrm{CO}_{2}\right)$ lasers in human skin in vivo. Photoaged forearm skin was treated using fully ablative (traditional) or fractionated $\mathrm{CO}_{2}$ laser. Skin samples were obtained at the indicated times after treatment and analyzed using immunohistology for (A) MMP-1, (B) MMP-3, and (C) MMP-9. Representative images from six subjects are shown.

(A)

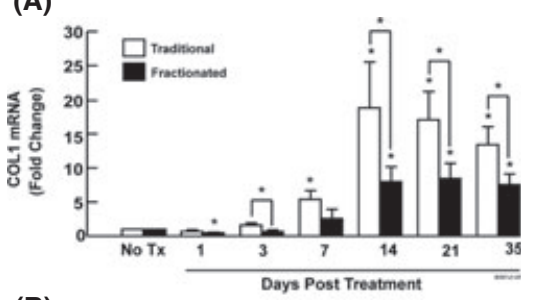

(B)

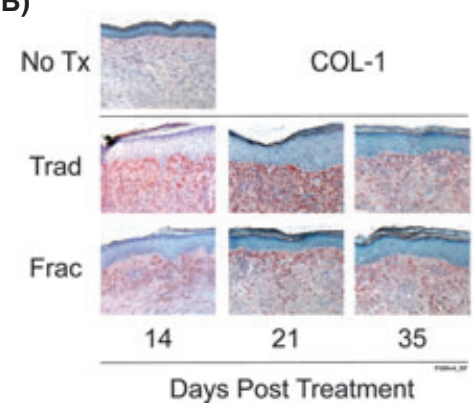

(C)

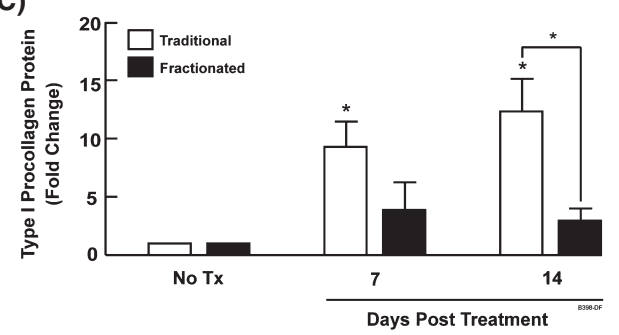

(D)

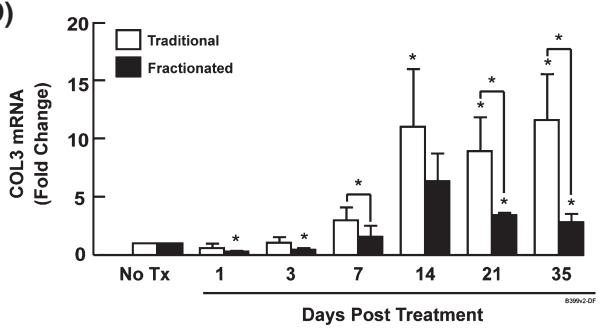

Figure 5. Induction of collagen gene and protein expression by fully ablative and fractionated carbon dioxide $\left(\mathrm{CO}_{2}\right)$ lasers in human skin in vivo. Photoaged forearm skin was treated with fully ablative (traditional) or fractionated $\mathrm{CO}_{2}$ laser. Skin samples were obtained at the indicated times post treatment. Data are means \pm standard errors of the mean (SEM), $* p<.05$. (A) Type I collagen gene expression measured using real-time polymerase chain reaction ( $N=6-10)$. (B) Type I procollagen protein expression measured using immunohistology. Representative images from six subjects are shown. (C) Type I procollagen protein quantified using enzyme-liked immunosorbent assay $(N=3)$. (D) Type III collagen gene expression measured by real-time PCR $(N=6-10)$.

protein expression after fully ablative or fractionated $\mathrm{CO}_{2}$ laser resurfacing. Increased staining as compared to baseline was observed within fibroblasts in the upper dermis at 2, 3, and 5 weeks after treatment (Figure 5B). Type I procollagen staining was generally stronger after fully ablative $\mathrm{CO}_{2}$ laser treatment than after fractionated $\mathrm{CO}_{2}$ laser treatment at all time points. To better assess this difference, we used an ELISA-based assay to quantify type I procollagen protein levels at 3 and 5 weeks after $\mathrm{CO}_{2}$ laser resurfacing (Figure 5C).

Average peak induction of type I procollagen protein was greater after fully ablative laser resurfacing (10.8-fold) than after fractionated laser treatment (3.4-fold).

Similar to type I procollagen, both types of $\mathrm{CO}_{2}$ laser resurfacing induced type III procollagen mRNA 
(A)

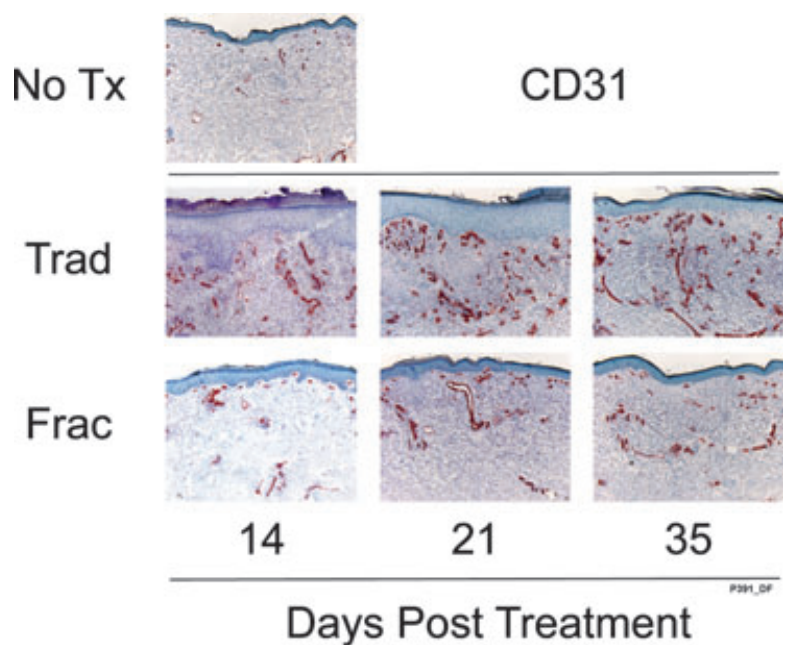

(B)

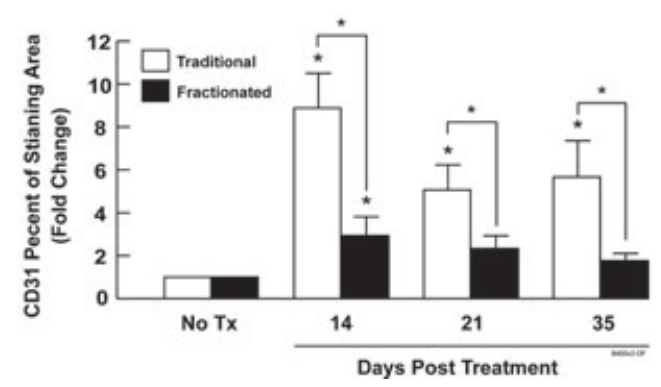

Figure 6. Increased vascularization after fully ablative and fractionated carbon dioxide $\left(\mathrm{CO}_{2}\right)$ laser treatment in human skin in vivo. Photoaged forearm skin was treated with fully ablative (traditional) or fractionated $\mathrm{CO}_{2}$ laser. Skin samples were obtained at the indicated times after treatment and analyzed for vascularization using immunohistology for the endothelial cell marker CD31. (A) Representative images of CD31 immunostaining from six subjects are shown. (B) The relative amount of CD31 immunostaining was quantified by computerized image analysis. Data are means \pm standard errors of the mean, $N=6-10, * p<0.05$.

(Figure 5D). Induction was observed at 2, 3, and 5 weeks after treatment. Average induction of type III procollagen mRNA was 2.5 times as great after fully ablative (10.5-fold) than after fractionated (4.2-fold) $\mathrm{CO}_{2}$ laser treatment.

In addition to production of new collagen fibrils in the ECM, dermal wound repair involves increased vascularization. ${ }^{21}$ We assessed dermal blood vessels in the upper dermis of skin sections by immunostaining with an antibody that recognizes the endothelial cell marker CD31. In sections from untreated skin, CD31-positive endothelial cells were associated with blood vessels, which appeared circular or elongated, some with lumens, depending on orientation within the dermis (Figure 6A). CD31-positive vessel structures were widely spaced apart. Fully ablative and fractionated $\mathrm{CO}_{2}$ laser treatment increased the density of CD31-positive vessels at 3 and 5 weeks after laser treatment from baseline (Figure 6A). Staining was particularly evident within the newly formed collagenous ECM in the upper dermis. Computerized image analysis revealed that both types of lasers increased CD31 staining. Average induction was 2.8 times as great after fully ablative (6.5-fold) than after fractionated (2.3-fold) $\mathrm{CO}_{2}$ laser treatment (Figure 6B).

\section{Discussion}

Fractionated ablative laser resurfacing (FLR) has been demonstrated to have substantial efficacy in the treatment of a variety of textural problems in human skin, including scars and rhytides secondary to photodamage. ${ }^{4,6,22}$ In addition, research using high-resolution ultrasound indicates that FLR results in increases in skin thickness. ${ }^{23} \mathrm{~A}$ study by Weiss and colleagues used a threedimensional optical profiling system to produce high-resolution topographical representations of treated atrophic scars. Mean scar volumes and maximal scar depths were calculated before and after FLR, and these parameters were shown to have improved $38.0 \%$ and $35.6 \%$, respectively, after treatment. ${ }^{24}$

Prignano and colleagues used immunohistochemical techniques to examine the cytokine milieu before and after fractionated ablative $\mathrm{CO}_{2}$ laser resurfacing in photodamaged human skin. ${ }^{25}$ Although this study was semiquantitative in nature (using a scale of $0-3$ to signify the level of perceived immunoreactivity), the authors demonstrated changes in immunostaining of some important cytokines and growth factors, including platelet-derived growth factor, endothelial growth factor, and transforming growth factor beta, 
after FLR. These changes appeared to be fluence dependent. This work supports the notion that fractionated laser resurfacing induces a controlled wound healing response. Similar research based on immunohistochemistry by Helbig and colleagues demonstrated that the microscopic wounds induced by FLR initially heal through regrowth of the epidermis followed by collagen production in the dermis. ${ }^{26}$ An inflammatory infiltrate including CD3+ and CD20+ cells appeared to be important in clearance of thermally altered tissue as dermal remodeling occurred.

Prior work by our group has revealed that a number of lasers and other aesthetic interventions may induce dermal remodeling to varying degrees. ${ }^{8-17}$ This research has resulted in a paradigm that indicates that the degree of clinical improvement in the texture of treated photodamaged skin produced using a given treatment may be estimated according to the quantified molecular changes it produces. It is clinically evident that fully ablative and fractionated ablative laser resurfacing may result in substantial aesthetic improvements, not only with respect to parameters such as dyspigmentation, but also in terms of textural smoothing of sun-damaged skin. It is also apparent that, with current technology and typical treatment parameters, fully ablative laser therapy produces more-profound changes. Despite subjective attempts to compare the results of these treatment approaches, what has been less clear is the degree to which improvements in skin texture based on FLR might approach those resulting from fully ablative resurfacing. With collagen I induction serving as perhaps the most direct predictor of clinical efficacy, the current data suggest that FLR may be expected to result in improvements in texture that are roughly $40 \%$ to $50 \%$ of those produced by fully ablative laser resurfacing after a single treatment.

It is possible that the more-rapid return to baseline of markers of dermal remodeling in fractionated lasertreated skin reflects the relatively quicker reepithelialization associated with that treatment modality.
Reepithelialization of the epidermis may act to downregulate the wound healing cascade, and this may be the focus of future research. Whether it is specifically more-rapid healing or simply a lower level of initial wounding and thus skin damage after FLR that facilitates the quicker return to baseline of these important biomarkers is speculative at this time.

The current data are indicative of changes that result from a single treatment, and it is possible that serial fractionated therapy might ultimately produce improvements that even more closely mimic changes seen after fully ablative laser resurfacing. The matter is the subject of future research by our group. Improvements with respect to other measurements of skin quality, including uniformity of skin color after FLR, were not measured in the current study and may prove to resemble those resulting from fully ablative resurfacing even more closely. One additional limitation of our study is that it involved sun-damaged forearm skin rather than facial skin, although we believe that the fundamental wound healing and dermal remodeling processes discussed above do not differ greatly between these two sites. Mitigating this is that our data are comparative in nature between the two laser techniques studied, and thus we would expect the relative results to be similar whether the skin studied was on the face or forearm. In addition, although the data reported here are indicative of results with the use of specific treatment parameters, the laser settings used in this study were selected to be generally within the range of those typically employed in a clinical setting.

We would hope that the information provided by our results will be useful for clinicians in discussions with patients regarding expected clinical outcomes with fractionated ablative laser resurfacing. As treatment parameters are optimized and the molecular and clinical changes of serial fractionated ablative laser therapy are measured, it is possible that we will be able to provide treatments with even greater clinical efficacy while maintaining the outstanding safety profile of this treatment approach. 
Acknowledgments The authors wish to thank Heather Chubb for her assistance with the statistical analysis of our data. We are also grateful to Suzan Rehbine, LPN, and Heather Kovarik, LPN, for their assistance with patient recruitment and treatment. We also thank Diane Fiolek for her assistance with the production of figures and for administrative support and the American Society for Dermatologic Surgery for supporting this work with a Cutting Edge Research Grant.

\section{References}

1. Ratner D, Tse Y, Marchell N, Goldman MP, et al. Cutaneous laser resurfacing. J Am Acad Dermatol 1999;1(3 Pt 1):365-89.

2. Nanni C, Alster T. Complications of carbon dioxide laser resurfacing-An evaluation of 500 patients. Dermatol Surg 1998;24:315-20.

3. Manstein D, Herron GS, Sink RK, Tanner H, et al. Fractional photothermolysis: a new concept for cutaneous remodeling using microscopic patterns of thermal injury. Lasers Surg Med 2004;34:426-38.

4. Ciocon DH, Engelman DE, Hussain M, Goldberg DJ. A split-face comparison of two ablative fractional carbon dioxide lasers for the treatment of photodamaged facial skin. Dermatol Surg 2011;37:784-90.

5. Cohen SR, Henssler C, Johnston J. Fractional photothermolysis for skin rejuvenation. Plast Reconstr Surg 2009;124:281-90.

6. Lapidoth M, Yagima Odo ME, Odo LM. Novel use of erbium: YAG (2,940-nm) laser for fractional ablative photothermolysis in the treatment of photodamaged facial skin: a pilot study. Dermatol Surg 2008;34:1048-53.

7. Chapas AM, Brightman L, Sukal S, Hale E, et al. Successful treatment of acneiform scarring with $\mathrm{CO}_{2}$ ablative fractional resurfacing. Lasers Surg Med 2008;40:381-6.

8. Karimipour DJ, Kang S, Johnson TM, Orringer JS, et al. Microdermabrasion: a molecular analysis following a single treatment. J Am Acad Dermatol 2005;52:215-23.

9. Karimipour DJ, Kang S, Johnson TM, Orringer JS, et al. Microdermabrasion with and without aluminum oxide crystal abrasion: a comparative molecular analysis of dermal remodeling. J Am Acad Dermatol 2006;54:405-10.

10. Karimipour DJ, Rittie L, Hammerberg C, Min VK, et al. Molecular analysis of aggressive microdermabrasion in photoaged skin. Arch Dermatol 2009;145:1114-22.

11. Orringer J, Voorhees J, Hamilton T, Hammerberg C, et al. Dermal matrix remodeling after nonablative laser therapy. J Am Acad Dermatol 2005;53:775-82.

12. Orringer JS, Hammerberg C, Hamilton T, Johnson TM, et al. Molecular effects of photodynamic therapy for photoaging. Arch Dermatol 2008;144:1296-302.
13. Orringer JS, Kang S, Johnson TM, Karimipour DJ, et al. Connective tissue remodeling induced by carbon dioxide laser resurfacing of photodamaged human skin. Arch Dermatol 2004;140:1326-32.

14. Sachs D, Kang S, Hammerberg C, Helfrich Y, et al. Topical 5fluorouracil improves actinic keratoses and photoaging: a clinical and molecular analysis. Arch Dermatol 2009;145:659-66.

15. Wang F, Garza LA, Kang S, Varani J, et al. In vivo stimulation of de novo collagen production caused by cross-linked hyaluronic acid dermal filler injections in photodamaged human skin. Arch Dermatol 2007;143:155-63.

16. Orringer JS, Rittie L, Baker D, Voorhees JJ, et al. Molecular mechanisms of nonablative fractionated laser resurfacing. $\mathrm{Br} \mathrm{J}$ Dermatol 2010;163:757-68.

17. Orringer JS, Rittie L, Hamilton T, Karimipour DJ, et al. Intraepidermal erbium:YAG laser resurfacing: impact on the dermal matrix. J Am Acad Dermatol 2011;64:119-28.

18. Rittié L, Kang S, Voorhees JJ, Fisher GJ. Induction of collagen by estradiol: difference between sun-protected and photodamaged human skin in vivo. Arch Dermatol 2008;144:1129-40.

19. Singer AJ, Clark RA. Cutaneous wound healing. N Engl J Med 1999;341:738-46.

20. Quan T, Qin Z, Xia W, Shao Y, et al. Matrix-degrading metalloproteinases in photoaging. J Investig Dermatol Symp Proc 2009;14:20-4.

21. Mahdavian Delavary B, van der Veer WM, van Egmond M, Niessen FB, et al. Macrophages in skin injury and repair. Immunobiology 2011;216:753-62.

22. Cervelli V, Gentile P, Spallone D, Nicoli F, et al. Ultrapulsed fractional $\mathrm{CO}_{2}$ laser for the treatment of post-traumatic and pathological scars. J Drugs Dermatol 2010;9:1328-31.

23. Tierney EP, Hanke CW, Petersen J, Bartley T, et al. Clinical and echographic analysis of ablative fractionated carbon dioxide laser in the treatment of photodamaged facial skin. Dermatol Surg 2010;36:2009-21.

24. Weiss ET, Chapas A, Brightman L, Hunzeker C, et al. Successful treatment of atrophic postoperative and traumatic scarring with carbon dioxide ablative fractional resurfacing: quantitative volumetric scar improvement. Arch Dermatol 2010;146:133-40.

25. Prignano F, Campolmi P, Bonan P, Ricceri F, et al. Fractional $\mathrm{CO}_{2}$ laser: a novel therapeutic device upon photobiomodulation of tissue remodeling and cytokine pathway of tissue repair. Dermatol Ther 2009; Nov (22 Suppl 1):S8-15.

26. Helbig D, Bodendorf MO, Grunewald S, Kendler M, et al. Immunohistochemical investigation of wound healing in response to fractional photothermolysis. J Biomed Opt 2009;14:064044.

Address correspondence and reprint request to: Jeffrey S. Orringer, MD, University of Michigan Medical School, 1500 E. Medical Center Drive, Department of Dermatology, 1910 Taubman Center, Ann Arbor, MI 48109, or e-mail: jorringe@umich.edu 